\title{
A Combined ESPRIT-MUSIC Method for FDA-MIMO Radar with Extended Range Ambiguity Using Staggered Frequency Increment
}

\author{
Yuwei Song, Guimei Zheng $(\mathbb{D}$, and Guoping Hu $\mathbb{D}$ \\ Air and Missile Defense College, Air Force Engineering University, Xi'an 710051, China \\ Correspondence should be addressed to Guimei Zheng; zheng-gm@163.com
}

Received 20 September 2018; Revised 12 March 2019; Accepted 16 April 2019; Published 7 July 2019

Academic Editor: Hervé Aubert

Copyright (C) 2019 Yuwei Song et al. This is an open access article distributed under the Creative Commons Attribution License, which permits unrestricted use, distribution, and reproduction in any medium, provided the original work is properly cited.

For the problem of joint angle and range estimation with frequency diverse array (FDA), MIMO radar, staggered frequency increment is proposed to expand the range ambiguity and the joint algorithm of ESPRIT and MUSIC is proposed to reduce the computational complexity. The uniformly weighted beampattern of FDA is a SINC-like function. Therefore, the grating lobe of range estimation exists. In this paper, staggered frequency increment is proposed to increase the distance of adjacent grating lobes. The proposed joint estimation algorithm firstly estimates the angle by using ESPRIT algorithm. Then we get the range estimation by MUSIC one-dimensional range search using the above estimated angle. In simulation results section, it is indicated in simulation results that the proposed method improves the range grating lobe and reduces the complexity.

\section{Introduction}

Frequency diverse array (FDA) has many potential advantages in radar applications [1-5]. The concept of FDA is described in [1] for the first time. References [2,3] summarize the development of FDA. References [4] and [5], respectively, address an important issue, decoupling of the slant range and spatial angle of FDA, by use of random carrier frequency and prime-carrier frequency. Its beampattern not only is decided by the spatial angle, but also is dependent on the target's slant range. Therefore, it has its own unique advantages in target antijamming and parameter estimation. In addition, multiple input and multiple output (MIMO) radar can provide the waveform diversity advantage. So, the FDA-MIMO radar has received a wide attention [6-9]. One of the important problems in FDA-MIMO radar is still the design of rangeindependent transmitted waveforms. The joint estimation of angle and slant range is an important topic for FDA-MIMO radar $[10,11]$. Reference [10] uses the subaperture synthesis method to solve the joint estimation of angle and range. Reference [11] proposes a method of transmitting two pulses to solve the joint estimation of angle and range, without optimizing the carrier frequency. It is a simple but practical.
For simplicity, slant range is expressed directly as range in the rest of the paper.

The uniformly weighted beampattern of FDA is a SINClike function. Therefore, it forms grating lobe for the range and angle estimation; that is, the range and angle estimation is ambiguous. For FDA-MIMO radar, the ambiguous of angle estimation disappears because of the received array in MIMO. But the grating lobe of range estimation exists.

On the other hand, generally speaking, joint estimation of angle and range needs to search in the two range-angle dimensions. Reference [11] proposes the use of a double transmitted pulses method, which only needs one-dimensional searching. It can greatly reduce the computational complexity. For further reducing the complexity, a joint estimation algorithm of ESPRIT and MUSIC is proposed in this paper. First, ESPRIT algorithm is used to estimate the angle. Then the range estimation is obtained by MUSIC one-dimensional searching using the above estimated angle. The angle and range estimations are automatically paired. The calculation of this method is smaller than that of [11].

Overall, there are two main contributions in this paper. First, we use the staggered frequency increment to expand the ambiguous range in the slant range estimation. Second, we 
use joint ESPRIT and MUSIC estimation algorithm to reduce the computational complexity.

Notations are as follows: Superscripts $(\cdot)^{T}$ and $(\cdot)^{H}$ denote complex transpose and conjugate transpose, respectively. $\otimes$ denotes Kronecker product. $\boldsymbol{I}_{M}$ is an $M \times M$ identity matrix. $\mathbf{0}_{(M-1) \times 1} \operatorname{diag}[\cdot]$ denotes diagonalization. $\angle(\cdot)$ denotes the angle of the complex value, and the range is from $-\pi$ to $\pi$.

\section{Signal Model}

Here we give a sketch of FDA-MIMO radar with one single target in Figure 1. In Figure $1, r$ is the target's range, $\theta$ denotes the source's angle, and $f_{0}, \Delta f, d, M$ denote reference carrier frequency, frequency increment, distance between the adjacent elements, and number of elements, respectively.

It is well known that when the transmit array is uniformly weighted, the transmitted beampattern can be expressed as [2]

$$
\begin{aligned}
& |A F(r, \theta)| \\
& \quad=\frac{\sin \left[M \pi\left(-\Delta f r / c+f_{0} d \sin \theta / c+\Delta f d \sin \theta / c\right)\right]}{\sin \left[\pi\left(-\Delta f r / c+f_{0} d \sin \theta / c+\Delta f d \sin \theta / c\right)\right]}
\end{aligned}
$$

where $c$ denotes light speed. This is a SINC-like function, and it is obvious that grating lobe will appear at $\Delta \mathrm{fr} / \mathrm{c}+$ $d f_{0} \sin \theta / c+d \Delta f \sin \theta / c=k, k=0, \pm 1, \pm 2, \ldots$. Therefore, it forms grating lobe for the range and angle estimation; that is, the range and angle estimation is ambiguous. For FDAMIMO radar, the ambiguity of angle estimation disappeared because of the received array in MIMO. However, the range estimation is still ambiguous.

We know that the range resolution in radar system is equal to $c / 2(M-1) \Delta f$ [11]. For the fixed size of array, the frequency increment $\Delta f$ will be very large if we need high range resolution. For large $\Delta f$, the range ambiguity caused by the beampattern will become very close. For example, range resolution is $150 \mathrm{~m}$ with a 21 -sensor meter-wave radar; it needs $1 \mathrm{M} \mathrm{Hz}$ bandwidth. Then the frequency increment $\Delta f$ is equal to $50 \mathrm{~K} \mathrm{~Hz}$. We set the target's angle $\theta=0^{\circ}$; we have $\Delta f r / c=k, k=0, \pm 1, \pm 2, \ldots$; it implies range ambiguity will appear in $6 \mathrm{~km}$ periodically. In this paper, we propose the use of staggered frequency increment to expand the ambiguity range of range estimation, in particular, the array transmits pulses with different frequency increments.

For an $M$-element FDA, the carrier frequency of each element is

$$
f_{m}=f_{0}+m \Delta f, \quad m=0, \cdots, M-1
$$

Uniform linear array (ULA) in both the transmitted and received arrays is considered in our paper. Then according to [11], we know that the transmitted steering vector equals

$$
\begin{aligned}
\boldsymbol{a}_{t} & (r, \theta) \\
\quad & =\left[1, \cdots, e^{\left(-j 2 \pi(M-1) \cdot\left(-\Delta f r / c+f_{0} d \sin \theta / c+\Delta f d \sin \theta / c\right)\right)}\right]
\end{aligned}
$$

$c$ is the speed of light, which is a constant. It is noted that the transmitted steering vector is dependent on the two

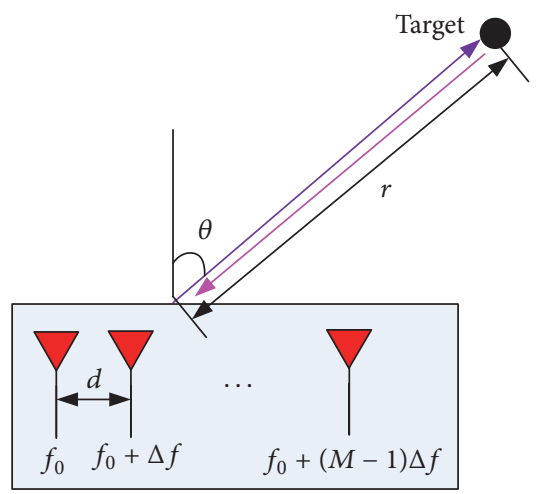

FIGURE 1: Sketch of FDA-MIMO radar with one single target.

dimensions of angle and range. Assuming that the MIMO radar is collocated, the received steering vector equals

$$
\boldsymbol{a}_{r}(\theta)=\left[1, e^{-j 2 \pi d \sin \theta / \lambda}, \cdots, e^{-j 2 \pi d \sin \theta(M-1) / \lambda}\right]^{T}
$$

Then the whole steering vector of the FDA-MIMO radar can be written as

$$
\boldsymbol{a}(r, \theta)=\boldsymbol{a}_{t}(r, \theta) \otimes \boldsymbol{a}_{r}(\theta)
$$

The received data of the FDA-MIMO radar can be expressed as

$$
\boldsymbol{x}(t)=\sum_{k=1}^{K} \boldsymbol{a}\left(r_{k}, \theta_{k}\right) s_{k}(t)+\boldsymbol{n}(t)=\boldsymbol{A s}(t)+\boldsymbol{n}(t)
$$

where $K$ denotes the number of targets. Steering matrix equals $\boldsymbol{A}=\left[\boldsymbol{a}\left(r_{1}, \theta_{1}\right), \cdots, \boldsymbol{a}\left(r_{K}, \theta_{K}\right)\right] \in \mathbb{C}^{M^{2} \times K}$. The source vector equals $\boldsymbol{s}(t)=\left[s_{1}(t), \cdots, s_{K}(t)\right]^{T} \in \mathbb{C}^{K \times 1}$, and the sources are set as zero mean Gaussian random process. The noise vector $\boldsymbol{n}(t)$ is also set as complex Gaussian white noise. Then the purpose of this paper is to calculate the range and angle of the $K$ targets according to (6).

\section{A Joint Estimation Method of ESPRIT and MUSIC with Staggered Frequency Increment}

3.1. Staggered Frequency Increment for Range Disambiguation. It is well known that FDA-MIMO radar is ambiguous in range estimation, and its ambiguous value equals $\Delta f r / c+$ $d f_{0} \sin \theta / c+d \Delta f \sin \theta / c=k, k=0, \pm 1, \pm 2, \ldots$. In the field of clutter rejection, staggered frequency technology in Moving Target Indicator (MTI) is used to disambiguate the ambiguous range estimation caused by high repetition frequency [12]. Motivated by this, different frequency increments between pulses are used to disambiguate the ambiguous range estimation caused by high frequency increment. 
For simplicity, we take two frequency increments for example, as follows:

$$
\begin{aligned}
& \frac{\Delta f_{1} r_{\mathrm{amb} 1}}{c}+\frac{d f_{0} \sin \theta}{c}+\frac{d \Delta f_{1} \sin \theta}{c}= k_{1}, \\
& k_{1}=0, \pm 1, \pm 2, \ldots \\
& \frac{\Delta f_{2} r_{\mathrm{amb} 2}}{c}+\frac{d f_{0} \sin \theta}{c}+\frac{d \Delta f_{2} \sin \theta}{c}= k_{2}, \\
& k_{2}=0, \pm 1, \pm 2, \ldots
\end{aligned}
$$

where $\Delta f_{1}$ is the frequency increment of the first transmitted pulse. $\Delta f_{2}$ is the frequency increment of the second transmitted pulse. $r_{\mathrm{amb} 1}$ represents the maximum unambiguous value corresponding to $\Delta f_{1} \cdot r_{\mathrm{amb} 2}$ represents the maximum unambiguous value corresponding to $\Delta f_{2}$. Then the maximum unambiguous value of the two pulses is the overlapping part of $r_{\mathrm{amb} 1}$ and $r_{\mathrm{amb} 2}$; that is, $r_{\mathrm{amb}}=\min \operatorname{amb}\left\{r_{\mathrm{amb} 1}, r_{\mathrm{amb} 2}\right\}$, where $\min \operatorname{amb}\{x, y\}$ denotes the smallest part of the same value of sets $x$ and $y$.

From the above analysis, we can see that as long as $\Delta f_{1}$ and $\Delta f_{2}$ are not equal to 0 , the maximum unambiguous value $r_{\mathrm{amb}}$ can be extended. But we need to find a proper proportion of $\Delta f_{1}$ and $\Delta f_{2}$ to maximize it. According to MTI selection criteria, we know that setting $\Delta f_{1}$ and $\Delta f_{2}$ as a mutually prime relationship will maximize the value $r_{\mathrm{amb}}$. Here coprime-rule is analyzed in detail. It is difficult to analyze with (7) under the effect of unknown parameter $\theta$. Because the period of range ambiguous value has nothing to do with the value of the angle $\theta$, the $\theta$ is set as $0^{\circ}$. Then (7) is simplified into the following form:

$$
\begin{aligned}
& \frac{\Delta f_{1} r_{\mathrm{amb} 1}}{c}=k_{1}, \quad k_{1}=0, \pm 1, \pm 2, \ldots \\
& \frac{\Delta f_{2} r_{\mathrm{amb} 2}}{c}=k_{2}, \quad k_{2}=0, \pm 1, \pm 2, \ldots
\end{aligned}
$$

Equation (8) is solved easily:

$$
\begin{aligned}
r_{\mathrm{amb}} & =\min \mathrm{amb}\left\{r_{\mathrm{amb} 1}, r_{\mathrm{amb} 2}\right\} \\
& =\min \mathrm{amb}\left\{\frac{c k_{1}}{\Delta f_{1}}, \frac{c k_{2}}{\Delta f_{2}}\right\}
\end{aligned}
$$

For (9), the first periodically maximum unambiguous value is calculated with $k_{1}=k_{2}=1$. The ratio of $c / \Delta f_{1}$ and $c / \Delta f_{2}$ is selected as coprime relation. It implies that staggered ratio of $\Delta f_{1}$ and $\Delta f_{2}$ is selected as coprime-rule. Then, the maximum ambiguous value is equal to

$$
r_{\mathrm{amb}}=\min \mathrm{amb}\left\{\frac{c}{\Delta f_{1}}, \frac{c}{\Delta f_{2}}\right\}=\frac{c}{\Delta f_{1}} \cdot \frac{c}{\Delta f_{2}}
$$

To sum up, when using a single frequency increment, i.e., $\Delta f_{1}$, the maximum unambiguous range is $c / \Delta f_{1}$. When $\Delta f_{2}$ is used, the maximum unambiguous range is $c / \Delta f_{2}$. When proposed mutual-prime frequency increment is used, maximum unambiguous range becomes $r_{\mathrm{amb}}=c / \Delta f_{1} \cdot c / \Delta f_{2}$, which is much extended.
3.2. Joint Estimation of Angle and Range Based on ESPRIT and MUSIC. To make use of the ESPRIT algorithm, we have to figure out the rotational invariance of the received data $\boldsymbol{x}(t)$. Because of the range $r$ dependency, it is difficult to find its rotational invariance for the whole steering vector $\boldsymbol{a}(r, \theta)$. But for the received steering vector $\boldsymbol{a}_{r}(\theta)$ of ULA, it is easy to find the rotational invariance in the angle domain. Then the rotational invariance is extended to the whole steering vector. It is our main contribution in this subsection. In the following, the derivation in detail is given. We define $\boldsymbol{a}_{1}(r, \theta)$ as $\boldsymbol{a}_{1}(r, \theta) \triangleq \boldsymbol{a}_{t}(r, \theta) \otimes \boldsymbol{a}_{r, 1}(\theta)$, where $\boldsymbol{a}_{r, 1}(\theta)$ represents the steering vector of the first $M-1$ received elements; then $\boldsymbol{a}_{r, 1}(\theta)$ equals

$$
\boldsymbol{a}_{r, 1}(\theta)=\left[1, e^{-j 2 \pi d \sin \theta / \lambda}, \cdots, e^{-j 2 \pi d \sin \theta(M-2) / \lambda}\right]^{T}
$$

We define $\boldsymbol{a}_{2}(r, \theta)$ as $\boldsymbol{a}_{2}(r, \theta) \triangleq \boldsymbol{a}_{t}(r, \theta) \otimes \boldsymbol{a}_{r, 2}(\theta)$, where $\boldsymbol{a}_{r, 2}(\theta)$ represents the steering vector of the last $M-1$ received elements; then $\boldsymbol{a}_{r, 2}(\theta)$ equals

$$
\boldsymbol{a}_{r, 2}(\theta)=\left[e^{-j 2 \pi d \sin \theta / \lambda}, \cdots, e^{-j 2 \pi d \sin \theta(M-1) / \lambda}\right]^{T}
$$

For $\boldsymbol{a}_{1}(r, \theta)$ and $\boldsymbol{a}_{2}(r, \theta)$, there is the following rotational invariant relationship:

$$
\begin{aligned}
\boldsymbol{a}_{2}(r, \theta) & =\boldsymbol{a}_{t}(r, \theta) \otimes \boldsymbol{a}_{r, 2}(\theta) \\
& =\boldsymbol{a}_{t}(r, \theta) \otimes\left[\boldsymbol{a}_{r, 1}(\theta) e^{-j 2 \pi d \sin \theta / \lambda}\right] \\
& =\boldsymbol{a}_{1}(r, \theta) e^{-j 2 \pi d \sin \theta / \lambda}
\end{aligned}
$$

Equation (13) can be converted to

$$
\boldsymbol{J}_{2} \boldsymbol{a}(r, \theta)=e^{-j 2 \pi d \sin \theta / \lambda} \boldsymbol{J}_{1} \boldsymbol{a}(r, \theta)
$$

where the selection matrices equal $\boldsymbol{J}_{1}=\boldsymbol{I}_{M} \otimes\left[\begin{array}{ll}\boldsymbol{I}_{M} & \mathbf{0}_{(M-1) \times 1}\end{array}\right]$ and $\boldsymbol{J}_{2}=\boldsymbol{I}_{M} \otimes\left[\begin{array}{ll}\mathbf{0}_{(M-1) \times 1} & \boldsymbol{I}_{M}\end{array}\right]$. We can see that (14) describes a rotational invariance of the whole steering vector. Equation (14) can be expressed as matrix form with all $K$ targets, as follows.

$$
\begin{aligned}
\boldsymbol{J}_{2} \boldsymbol{A}(r, \theta) & =\boldsymbol{J}_{1} \boldsymbol{A}(r, \theta) \boldsymbol{\Theta}(\theta) \\
\boldsymbol{\Theta}(\theta) & \triangleq \operatorname{diag}\left[e^{-j 2 \pi d \sin \theta_{1} / \lambda}, \cdots, e^{-j 2 \pi d \sin \theta_{K} / \lambda}\right]
\end{aligned}
$$

It is noted that the matrix $\Theta(\theta)$ depends only on angle $\theta$, and the angle estimation can be calculated according to the rotational invariant relation of formula (15). Firstly, the received data in (6) is used to calculate the covariance matrix and then eigendecomposition is implemented to obtain its signal subspace and noise subspace. For the signal subspace, we have $\boldsymbol{E}_{S}=\boldsymbol{A T}, \boldsymbol{T}$ is a nonsingular matrix. Substituting it into formula (15), we have

$$
\boldsymbol{J}_{2} \boldsymbol{E}_{S}=\boldsymbol{J}_{1} \boldsymbol{E}_{S} \boldsymbol{\Psi}(\theta)
$$

where $\boldsymbol{\Psi}(\theta)=\boldsymbol{T}^{-1} \boldsymbol{\Phi}(\theta) \boldsymbol{T}$. Under the condition of noise, (16) can be solved by least square or total least square method; 


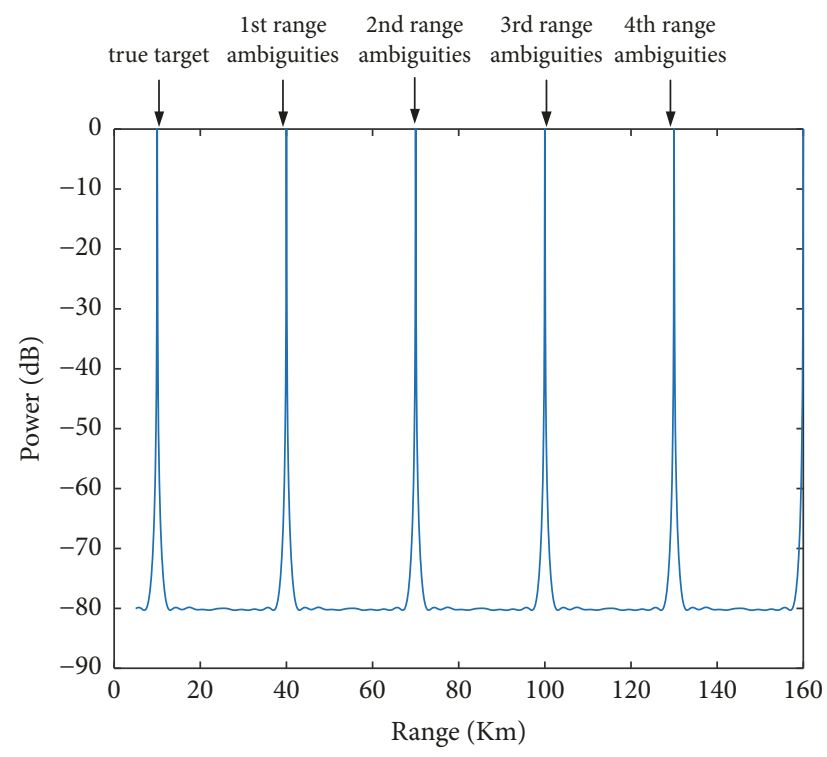

(a) Frequency increment is $10 \mathrm{kHz}$

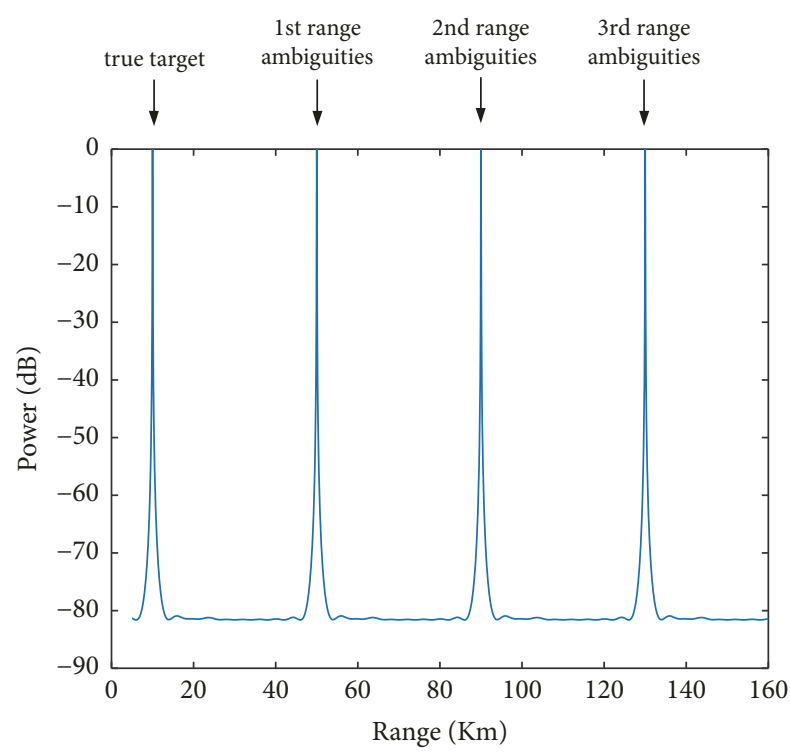

(b) Frequency increment is $7.5 \mathrm{kHz}$

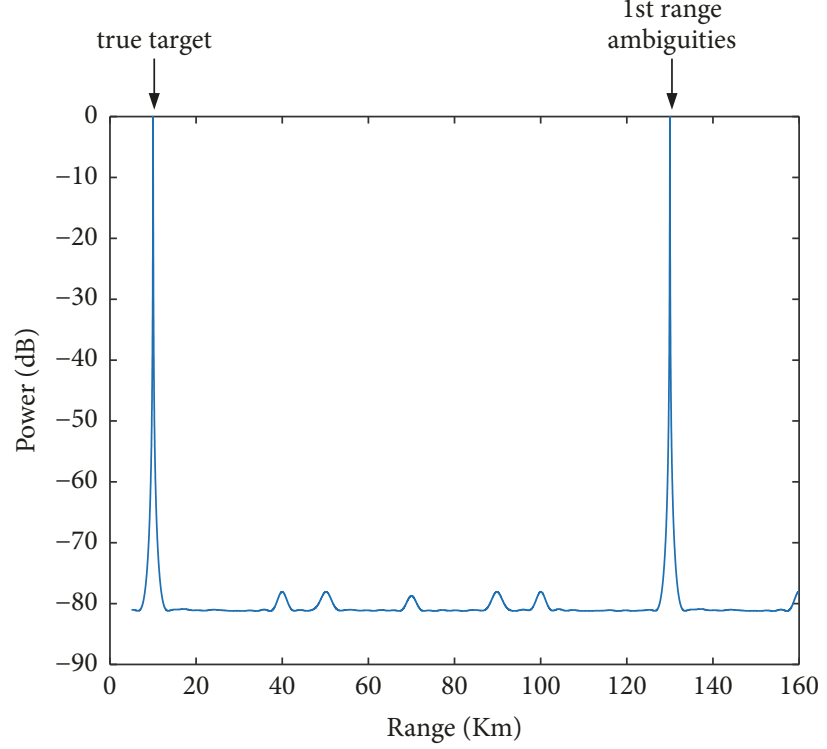

(c) Staggered frequency increment

FIGURE 2: The estimated results of range spectrum with first target.

then the estimation of $\Psi(\theta)$ is obtained. Therefore, the angle estimation $\hat{\theta}$ can be calculated by the following formula:

$$
\widehat{\theta}_{k}=-\arcsin \left\{\frac{\lambda}{2 \pi d} L\left([\Phi(\theta)]_{k k}\right)\right\}, \quad k=1, \cdots, K
$$

The diagonal elements $[\Phi(\theta)]_{k k}$ of the matrix $\Phi(\theta)$ are equal to the eigenvalues after the eigendecomposition with $\Psi(\theta)$. According to the signal model of Section 2, it is well known that the estimation of angle and range can be realized by the two-dimensional conventional MUSIC search algorithm:

$$
f_{2 \mathrm{D}-\mathrm{MUSIC}}(\theta, r)=\frac{1}{\boldsymbol{a}(\theta, r)^{H} \boldsymbol{E}_{N} \boldsymbol{E}_{N}^{H} \boldsymbol{a}(\theta, r)}
$$

The estimated angle $\hat{\theta}$ is used to avoid two-dimensional searching. Each estimated angle $\widehat{\theta}_{k}, k=1, \cdots, K$ is substituted into (18), and the range estimation can be obtained by the following one-dimensional search:

$$
\begin{array}{r}
\widehat{r}_{k}=\max \left[\frac{1}{\boldsymbol{a}\left(\hat{\theta}_{k}, r_{k}\right)^{H} \boldsymbol{E}_{N} \boldsymbol{E}_{N}^{H} \boldsymbol{a}\left(\hat{\theta}_{k}, r_{k}\right)}\right], \\
k=1, \cdots, K
\end{array}
$$

Note that the angle and range is automatically paired. The algorithm is performed by firstly finding the angle of the 


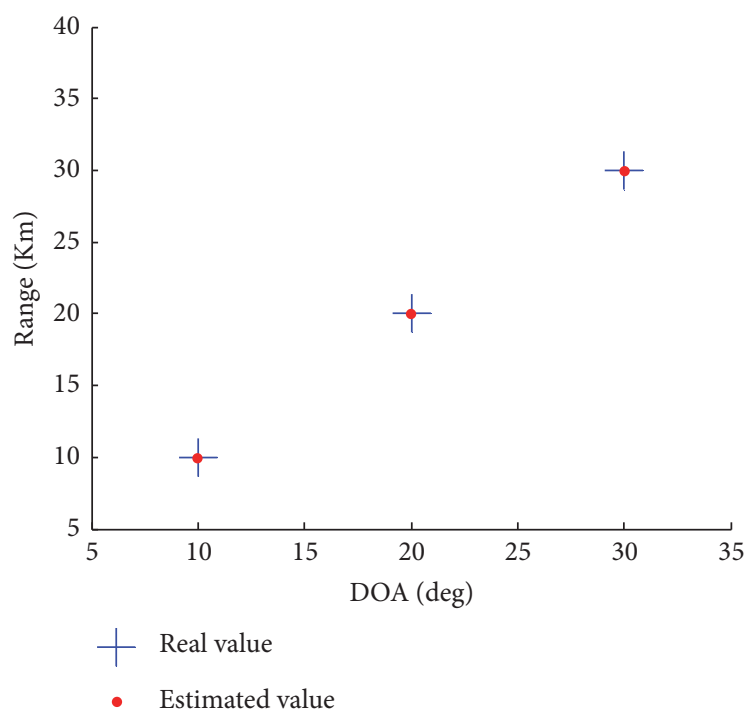

FIgURE 3: The estimated results of the angle and range.

target and secondly determining the range of target. The onedimensional search method requires $K$ times successive onedimensional search, which is the same as [11].

Note 1. This algorithm does not consider the range ambiguity caused by high pulse repetition frequency. The problem can be solved by the method of [13].

Note 2. In order to ensure that angle estimation is unambiguous, the large frequency increment is selected to compute the distance of the adjacent elements; that is, $d=c / 2[f+(M-$ 1) $\left.\max \left(\Delta f_{1}, \Delta f_{2}\right)\right]$.

\section{Simulation Results}

The number of elements is $M=10$. The same range bin has $K=3$ targets. Their angles are $\theta=\left[10^{\circ}, 20^{\circ}, 30^{\circ}\right]$. The ranges are set as $r=[10 \mathrm{~km}, 20 \mathrm{~km}, 30 \mathrm{~km}]$. The number of snapshots is $L=200$. The signal to noise ratio (SNR) is $\mathrm{SNR}=20 \mathrm{~dB}$. We set that the staggered frequency increment equals $10 \mathrm{kHz}$ and $7.5 \mathrm{kHz}$. Then the ratio $\Delta f_{1}: \Delta f_{2}=4: 3$, which is coprime relation.

If the staggered frequency increment is not used, the ambiguities corresponding to the two frequency increments are $30 \mathrm{~km}$ and $40 \mathrm{~km}$, respectively. The estimated range spectrum of the first target is given in the following. Figures 2(a), 2(b), and 2(c) give the results of the frequency increment of $10 \mathrm{kHz}, 7.5 \mathrm{kHz}$, and the proposed staggered frequency increment, respectively. The range estimation results of Figure 2(a) are $10 \mathrm{~km}, 40 \mathrm{~km}, 70 \mathrm{~km}, 100 \mathrm{~km}$, and $130 \mathrm{~km}$, corresponding to the true range of target, the 1st, $2 \mathrm{nd}$, 3rd, and 4 th range ambiguities. The peaks mean the target's estimation result. It uses a conventional approach with a frequency linear increment in one pulse. And the frequency increment is 10 $\mathrm{kHz}$. The range estimation results of Figure $2(\mathrm{~b})$ are $10 \mathrm{~km}$, $50 \mathrm{~km}, 90 \mathrm{~km}$, and $130 \mathrm{~km}$, corresponding to the true range of target, the 1st, $2 \mathrm{nd}$, and 3rd range ambiguities. It also uses the conventional approach with a frequency linear increment in one pulse. And the frequency increment is $7.5 \mathrm{kHz}$. The range estimation results of Figure 2 (c) are $10 \mathrm{~km}$ and $130 \mathrm{~km}$, corresponding to the true range of target and the range ambiguity. It is the result of use of staggered frequency increment. We can be seen that the range ambiguity in Figure $2(\mathrm{c})$ is $120 \mathrm{~km}$. These results are in full agreement with the results of our theoretical analysis. We can clearly see from Figure 2(c) that a small magnitude remains at a distance corresponding to kth range ambiguity of the target. In fact, the result of Figure 2(c) is equal to the extraction of the same peeks between Figures 2(a) and 2(b). This residual magnitude is so small that we can ignore. The result is similar to the filter of MTI [12].

The results of Figures 3, 4, and 5 are given for the angle and range of the three targets. And, of course, the results are within the unambiguous range. It can be seen from Figure 3 that the results of 100 Monte Carlo experiments are correct and the pairing results are right.

A RMSE comparison with [11] is given in Figure 4. For the fairness of comparison, we transform the DBF algorithm used in [11] to super resolution algorithm, that is, MUSIC algorithm based on one dimension search. We know that the estimation preference of MUSIC algorithm is better than that of the DBF algorithm under the case of the multiple targets. We can see that the method in [11] has slightly better estimation accuracy than that of the proposed algorithm.

We can see from (16) that the proposed algorithm does not use the full array aperture in the angle estimation step and uses only the aperture of the received array. Method in [11] uses (18), and we know that the full array aperture is used. That is why the method in [11] has better performance than that of the proposed algorithm.

Last, we give the comparison figure with running time. The range of range search is set as $5 \mathrm{~km}-35 \mathrm{~km}$, and the searching interval is $5 \mathrm{~m}$. For the proposed algorithm, we use two pulses and successive single pulse to compare the computational complexity with [11]. The range of angle search in [11] is -90 to 90 degrees, and the interval is set as three kinds: 0.1 degrees, 0.01 degrees, and 0.001 degrees. It is indicated in Figure 5 that if the successive single pulse is used, the computational time of our algorithm is smaller than that of [11]. When two pulses are used, the computational time is more than 0.1 degrees, less than 0.001 degrees, and equivalent almost to 0.01 degrees.

The proposed algorithm using successive single pulse means that one-pulse data with $10 \mathrm{kHz}$ frequency increment is used firstly, and then another pulse data with $7.5 \mathrm{kHz}$ frequency increment is used. The proposed algorithm using two pulses means that we set the two-pulse data into (6) together and then use (16) to estimate the angle.

\section{Conclusions}

The problem of the ambiguous range and angle estimation of FDA-MIMO radar has been investigated in this paper. The unambiguous range is extended by using the staggered frequency increment. ESPRIT algorithm is used to reduce 


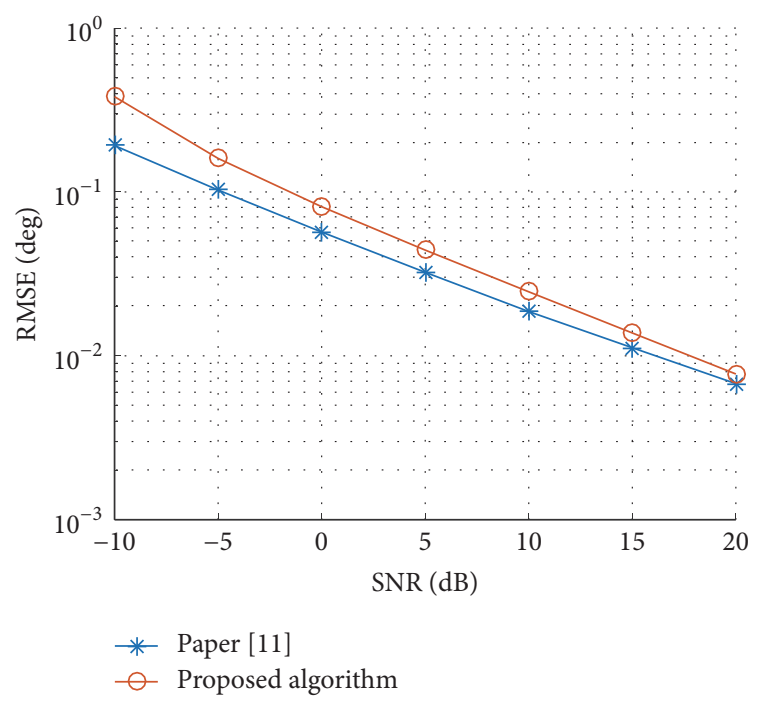

(a) Angle estimation

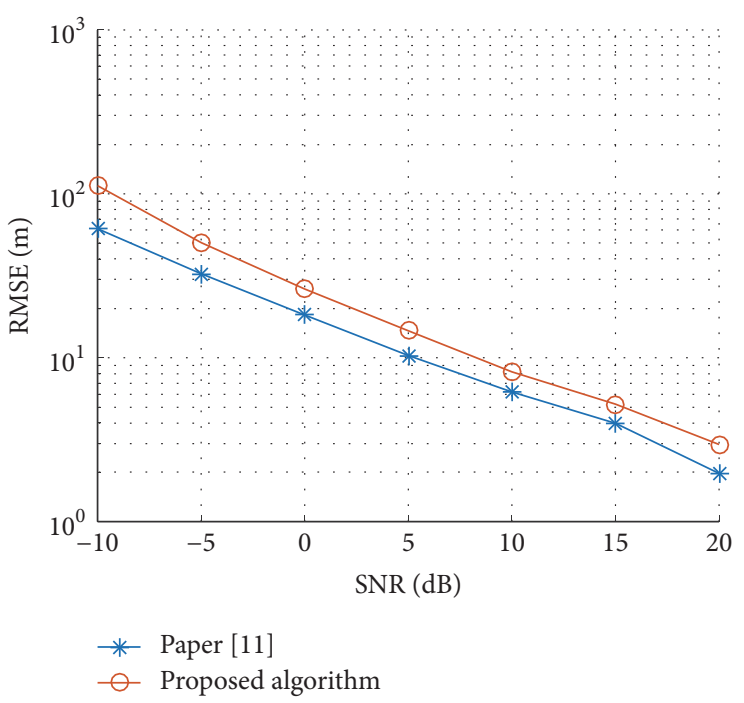

(b) Range estimation

FIGURE 4: RMSE estimation results.

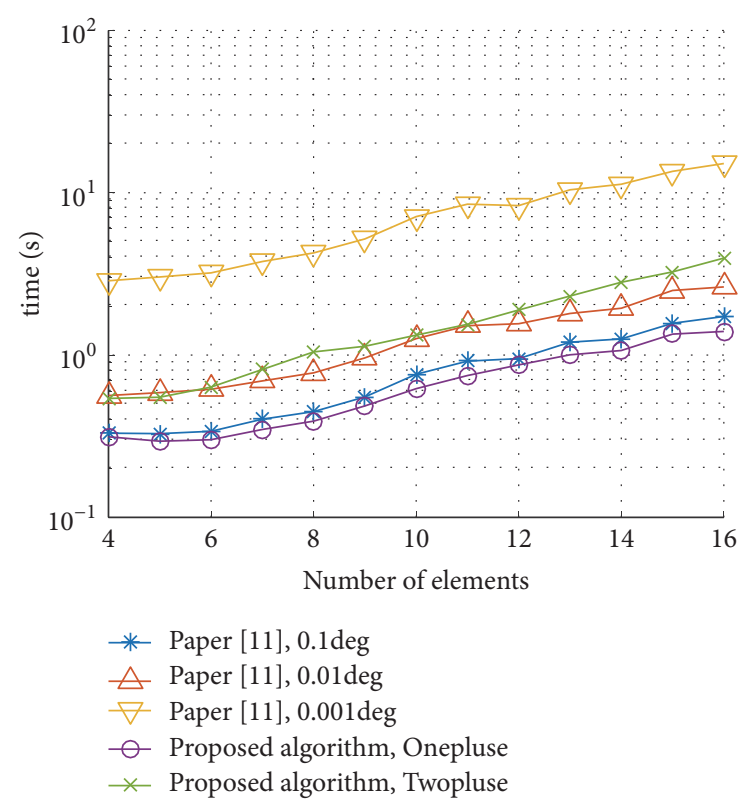

FiguRE 5: Comparison of computational complexity.

the computational complexity compared with angle search method. And our method can also get the result of automatic pairing of angle and range estimation.

We should know that two staggered frequency increments methods in our paper can be considered as the example of the $n$ staggered frequencies increments method. Unfortunately, we cannot give the design for $n$ staggered frequencies increments. It will be the focus of our future work. In addition, the use of the combination of ESPRIT and MUSIC can be also improved in the aspect of complexity.

\section{Data Availability}

The data used to support the findings of this study are included within the supplementary information file (available here).

\section{Conflicts of Interest}

The authors declare that they have no conflicts of interest.

\section{Acknowledgments}

This work was supported by National Natural Science Foundation of China under Grant 61601504.

\section{Supplementary Materials}

The resource of ESPRIT-MUSIC method for FDAMIMO radar with staggered frequency increment. (Supplementary Materials)

\section{References}

[1] P. Antonik, M. C. Wicks, H. D. Griffiths, and C. J. Baker, "Frequency diverse array radars," in Proceedings of the 2006 IEEE Conference on Radar, pp. 215-217, IEEE, Verona, NY, USA, 2006.

[2] W. Wang, "Frequency diverse array antenna: new opportunities," IEEE Antennas and Propagation Magazine, vol. 57, no. 2, pp. 145-152, 2015.

[3] A. Basit, W. Khan, S. Khan, and I. M. Qureshi, "Development of frequency diverse array radar technology: a review," IET Radar, Sonar \& Navigation, vol. 12, no. 2, pp. 165-175, 2018.

[4] Y. Liu, H. Ruan, L. Wang, and A. Nehorai, "The random frequency diverse array: a new antenna structure for uncoupled 
direction-range indication in active sensing," IEEE Journal of Selected Topics in Signal Processing, vol. 11, no. 2, pp. 295-308, 2017.

[5] S. Qin, Y. D. Zhang, M. G. Amin, and F. Gini, "Frequency diverse coprime arrays with coprime frequency offsets for multi-target localization," IEEE Journal of Selected Topics in Signal Processing, vol. 11, no. 2, pp. 321-335, 2017.

[6] W.-Q. Wang and H. Z. Shao, "A flexible phased-MIMO array antenna with transmit beamforming," International Journal of Antennas and Propagation, vol. 2012, Article ID 609598, 10 pages, 2012.

[7] P. F. Sammartino, C. J. Baker, and H. D. Griffiths, "Frequency diverse MIMO techniques for radar," IEEE Transactions on Aerospace and Electronic Systems, vol. 49, no. 1, pp. 201-222, 2013.

[8] W.-Q. Wang, "Phased-MIMO radar with frequency diversity for range-dependent beamforming," IEEE Sensors Journal, vol. 13, no. 4, pp. 1320-1328, 2013.

[9] K. Gao, W.-Q. Wang, and J. Cai, "Frequency diverse array and MIMO hybrid radar transmitter design via Cramer-Rao lower bound minimisation," IET Radar, Sonar \& Navigation, vol. 10, no. 9, pp. 1660-1670, 2017.

[10] W.-Q. Wang and H. C. So, "Transmit subaperturing for range and angle estimation in frequency diverse array radar," IEEE Transactions on Signal Processing, vol. 62, no. 8, pp. 2000-2011, 2014.

[11] W.-Q. Wang and H. Z. Shao, "Range-angle localization of targets by a double-pulse frequency diverse array radar," IEEE Journal of Selected Topics in Signal Processing, vol. 8, no. 1, pp. 1-9, 2014.

[12] M. I. Skolnik, Radar Handbook, The McGraw-Hill Companies Inc., New York, NY, USA, 3rd edition, 2008.

[13] J. W. Xu, G. S. Liao, S. H. Zhu, L. Huang, and H. C. So, "Joint range and angle estimation using MIMO radar with frequency diverse array," IEEE Transactions on Signal Processing, vol. 63, no. 13, pp. 3396-3410, 2015. 


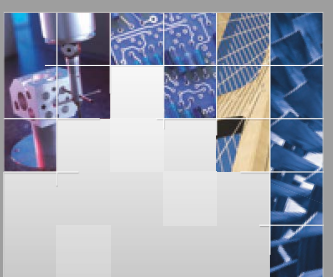

\section{Enfincering}
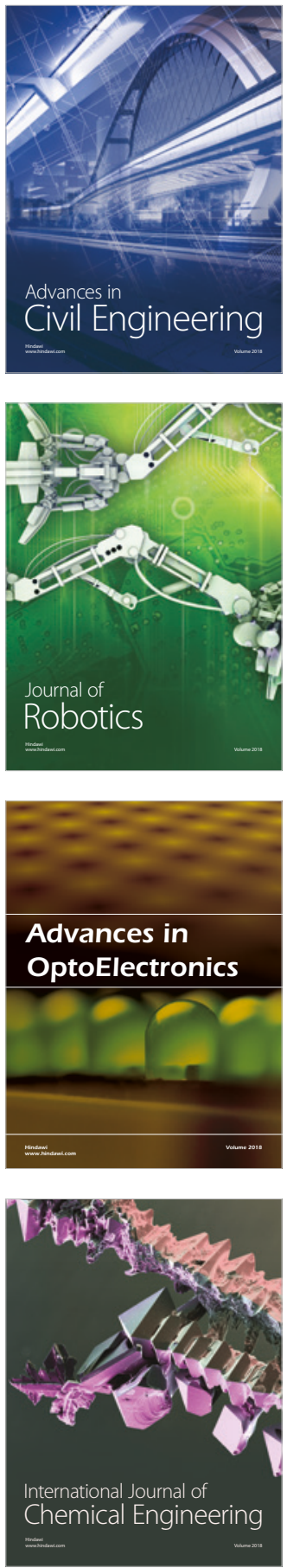

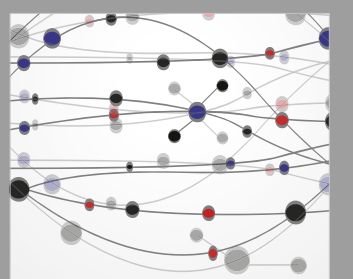

\section{Rotating \\ Machinery}

The Scientific World Journal

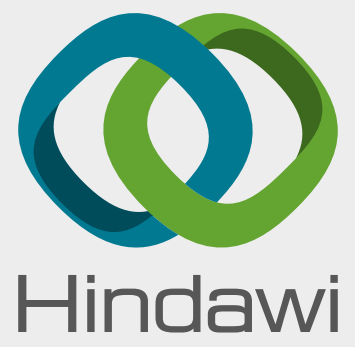

Submit your manuscripts at

www.hindawi.com
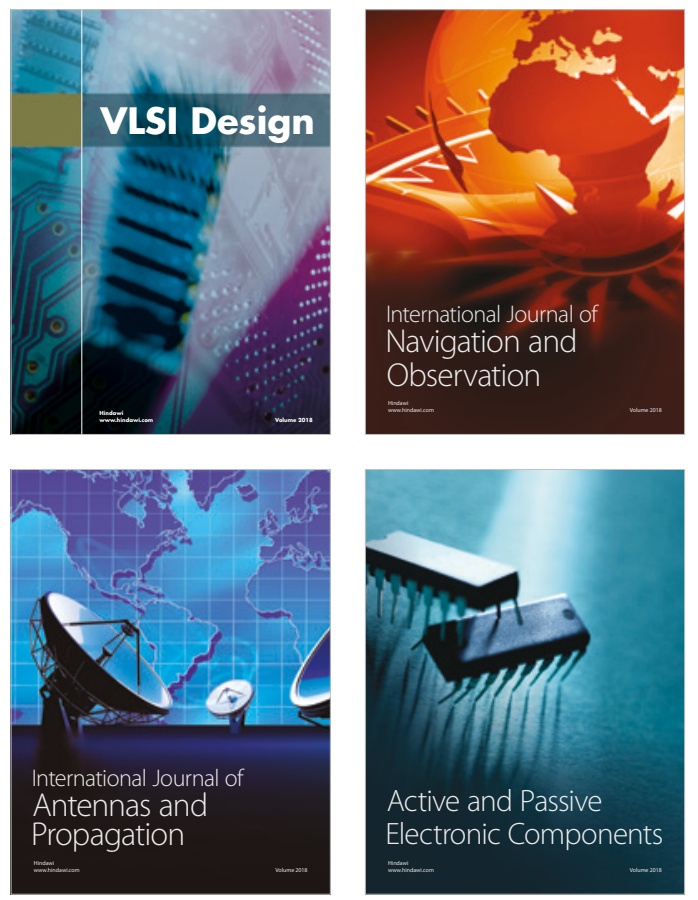
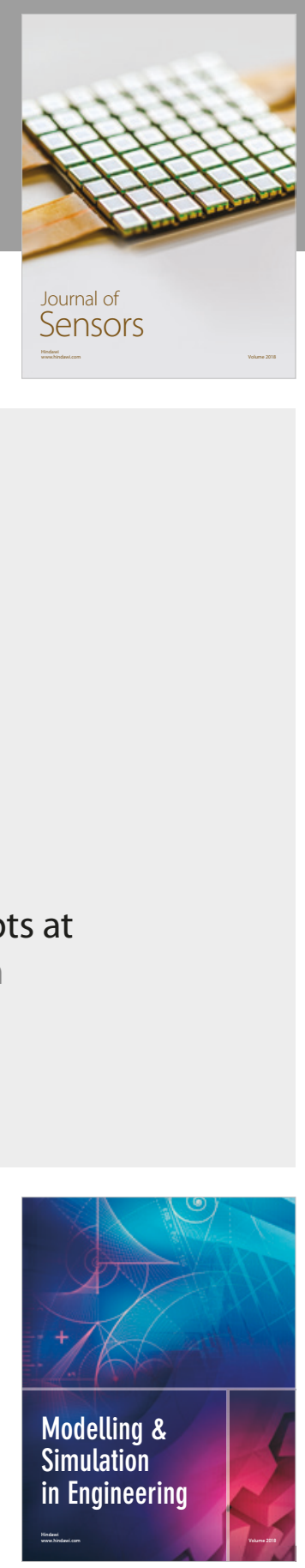

\section{Advances \\ Multimedia}
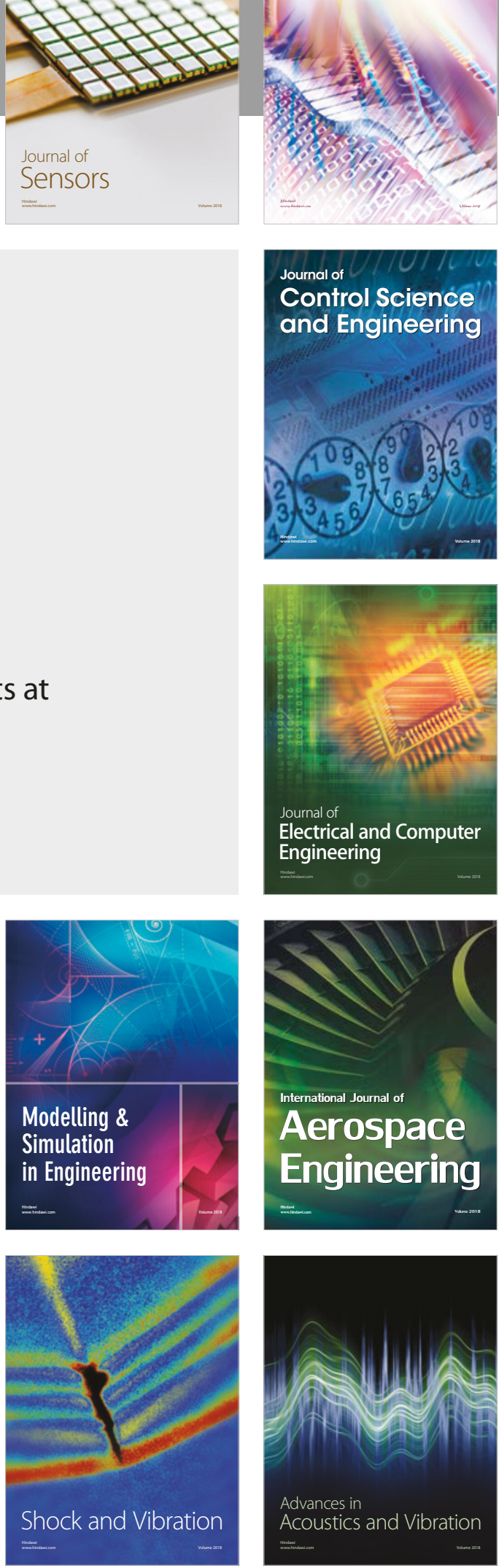